\title{
El perfil del docente investigador: hacia sus dimensiones y fortalecimiento
}

\section{The profile of the research teacher: towards its dimensions and its strengthening}

\author{
Salomón Vásquez-Villanueva ${ }^{1}$, Salomón Axel Vásquez Campos ${ }^{2}$, Carlos Alberto \\ Vásquez Villanueva ${ }^{3}$, Rodolfo Alania Pacovilca ${ }^{4}$, Moisés Díaz-Pinedo ${ }^{5}$, \\ Marina del Águila Gonzáles de Castillo ${ }^{6}$, \\ Universidad Peruana Unión $1,2,3,4,5$ \\ Universidad Nacional de la Amazonia Peruana ${ }^{6}$
}

iD ORCID ID: https://orcid.org/0000-0001-8824-6176

ORCID ID: https://orcid.org/0000-0001-9405-0794²

ORCID ID: https://orcid.org/0000-0002-0782-7364³

ORCID ID: https://orcid.org/0000-0001-9522-24714

ORCID ID: https://orcid.org/0000-0002-2106-99345

ORCID ID: https://orcid.org/0000-0003-2343-45336

Recibido: 03 de enero de 2020

Aceptado: 15 de junio de 2020

\begin{abstract}
Resumen
Se ha realizado el estudio con el doble objetivo: determinar las dimensiones del perfil del docente investigador y seleccionar los procedimientos para fortalecer el perfil referido. El perfil del docente investigador se ha constituido en un universo total vasto y complejo, polémico, incluso adverso en varios aspectos, en cuyo universo se plantean muchas preguntas quedando sin respuestas objetivas, clara, precisas, convincentes. En este contexto, se ha trabajado el perfil del docente investigador: sus dimensiones y procedimientos para fortalecerlos. Se concluye que el perfil del docente investigador presenta las siguientes dimensiones: pedagógica, académico profesional, inteligencia emocional, ético, publicación, permanencia y visibilidad. Además, se fortalece mediante la orientación del investigador experto, la legislación de los estados, la formación investigativa, la asistencia a eventos de investigación.
\end{abstract}

Palabras clave: perfil de profesor investigador, perfil pedagógico, perfil académico, perfil académico profesional.

${ }^{1}$ Correspondencia al autor:

E-mail: salomonv@upeu.edu.pe. 


\begin{abstract}
The study has been carried out with the double objective: to determine the dimensions of the profile of the research teacher and to select the procedures to strengthen the referred profile. The profile of the research professor has become a vast and complex universe, controversial, even adverse in several aspects, in which universe many questions are posed, leaving no objective, clear, precise, and convincing answers. In this context, the profile of the research teacher has been studied: its dimensions and procedures to strengthen them. It is concluded that the profile of the research teacher presents the following dimensions: pedagogical, professional academic, emotional intelligence, ethics, publication, permanence and visibility. In addition, it is strengthened through the guidance of the expert investigator, state legislation, investigative training, and attendance at research events.
\end{abstract}

Keywords: profile of the research teacher, pedagogical profile, professional academic profile.

\title{
Introducción
}

En torno del perfil del investigador, surgen ciertas cuestiones de las cuales no se puede eludir. ¿Cuál es el perfil del docente investigador? ¿Cuáles son las dimensiones del perfil del docente universitario? ¿Cómo fortalecer dicho perfil? ¿Dónde y cómo se forma el perfil en cuestión? ¿Quiénes lo forman? ¿Cuál es la función de este perfil? ¿Es único el perfil del investigador para todas las disciplinas del saber humano? Posiblemente queden al margen muchas cuestionas más; en este sentido, no se pretende abarcar todas, porque los intereses y la cosmovisión también resultan determinantes.

En una aproximación conceptual, el perfil "es complejo e integra actividades como coordinar proyectos de investigación, publicaciones periódicas, enseñar competencias de investigación a los estudiantes, impartir clases, llevar a cabo tutorías, gestión del proceso de enseñanza-aprendizaje y conocer los procesos de evaluación” (Álvarez et al., 2014, en Hernández-Herrera y Ramos-Mendoza, 2018, p. 7).

El perfil del docente investigador se ha magnificado en algunos casos; posiblemente, en otros, se ha trivializado, a pesar de las exigencias y las rigurosidades profesionales, académicas e investigativas, sin saber qué hacer ni dónde quedarse. Los estados, a través de sus respectivos órganos gubernamentales, han exigido-y exigen aun-a las universidades y a otras instituciones que afinen el perfil del docente investigador, incluso han generado normas con las que se facilita la construcción del perfil referido.

El lente no es el mismo para todas las personas y todas las universidades, que administran estas solicitudes y estos requerimientos; incluso dentro de una misma universidad, existen separaciones, alternativas disociadas, separatistas; el universo de la 
investigación y de las instituciones comprometidas se escinde, por los criterios, las políticas, los paradigmas, las concepciones, las experiencias, los cuales no son totalmente universales, ni convencionales. Es decir, el perfil del docente investigador se adminis tra, se enfoca desde múltiples perspectivas.

Por ello, el objetivo de este estudio es determinar las dimensiones del perfil del docente investigador; además seleccionar los procedimientos de respectivo fortalecimiento, con el propósito de darle mayor funcionalidad y efectividad en el proceso de investigación, dentro de las universidades.

\section{Perfil del docente investigador}

\section{Aproximaciones a las dimensiones del perfil del investigador}

No es lo mismo un "docente investigador" que un "investigador docente." Lo primero señala a un docente universitario que tiene formación, aprendizaje, preparación, ejercicio y experiencia en la investigación; el ejercicio y la exigencia de la docencia han obligado la investigación. En cambio, lo segundo implica alguien que "ha nacido investigador", luego ha ejercido la docencia; esta es consecuencia de la investigación. Definitivamente el docente investigador se forma en la universidad. Es un docente profesional de la ciencia, de la investigación, convive y vive para la docencia en la investigación. El docente investigador posee virtudes y desaciertos, no es perfecto; sin embargo, mejora todos los días merced a su motivación más intrínseca que extrínseca, a su interés construido por sí mismo, debido a su propio quehacer y su experiencia vital. El docente investigador desarrolla un determinado perfil profesional para sí y sus discípulos.

a) Perfil pedagógico

Para Canizales-Rivas y Vijil-Mejía (2015), un docente en la enseñanza de la investigación, necesita elaborar un marco teórico, fortalecer la operacionalización de las variables, desarrollar la experiencia docente en la elaboración de instrumentos, perfeccionar el anális is estadístico, mejorar la elaboración y presentación de los informes de investigación.

El docente investigador es un pedagogo de la investigación. Enseña la investigación recurriendo a lineamientos y principios didácticos. Es decir, quien enseña investigación debe mejorar y perfeccionar su didáctica, sus estrategias y su metodología pedagógica, 
con el propósito de entregar su enseñanza sistematizada, y así facilitar el aprendizaje de los estudiantes; el docente investigador, imbuido de estas condiciones, estimula y motiva la investigación, procesa su formación, y genera discípulos en esta disciplina. Estas apreciaciones, para conectarse al espíritu del estudio, permiten destacar que el docente investigador tiene un perfil pedagógico, ligado al proceso enseñanza aprendizaje de la investigación.

b) Perfil académico profesional

En este contexto, resulta muy interesante, pertinente y oportuno observar la interrogante de Rodriguez-Morales et al., (2016): “Cuál debería ser el perfil de quien se denomine investigador en Colombia y Perú?" Esta pregunta compromete a estos dos países hermanos, por eso reclama la atención de todos los investigadores. La investigación constituye proceso, función, y exigencia; pues el académico y profesional proyectan y dejan en el escenario de la investigación varias imágenes: institucional, personal, investigativa. No quedan al margen, no se sustraen; las universidades siempre forman y desarrollan al investigador; perfeccionan sus competencias y sus habilidades. La academia está en la universidad. La comunidad, los tiempos y las diversas necesidades demandan a las universidades una buena preparación, formación y desarrollo de los investigadores con un perfil académico profesional, que revele capacidad, competencias, habilidades, compromiso e identidad con la sociedad donde vive y a la cual debe servirle.

Por su parte, Hortigüela, González y Hernando (2017) conciben el perfil de investigador con el sentido de docente universitario, quien posee valores éticos y "perfil flexible y dinámico que le permita adaptarse a la diversidad y a los nuevos retos y demandas surgidas de la sociedad del conocimiento y la información" (p. 39). A este diseño de perfil se le atribuye algunos matices sustanciales; por ejemplo, la ética, la flexibilidad, el dinamismo, la diversidad, el conocimiento, la información, cuyo universo es admirable, envidiable e inevitable en el espacio seleccionado y clasificado, llamado universidad.

c) Perfil de inteligencia emocional

Hernández-Herrera y Ramos-Mendoza (2018) han realizado un estudio en el cual analizan la presencia de dos constructos: inteligencia emocional y prácticas docentes en los profesores investigadores. Se entiende que la inteligencia emocional facilita el razonamiento más eficiente, además produce una vida emocional más saludable. Pues quien tiene inteligencia emocional posee habilidades "para percibir y regular las 
emociones con el propósito de obtener un desarrollo emocional e intelectual altamente positivo". El docente investigador-debido a su supuesta inteligencia emocional-tiene habilidades y capacidades para percibir, identificar y expresar las emociones y los sentimientos que promuevan cambios y convencimientos. Es persuasivo y convincente.

d) El perfil ético

Según Hortigüela et al. (2017), el docente universitario está obligado a mantener "un perfil flexible y dinámico que le permita adaptarse a la diversidad y a los nuevos retos y demandas surgidas de la sociedad del conocimiento y la información” (p.39). Además, a mantener la ética expresada en la práctica llena de "honestidad y de integridad académica que han de regular la forma de hacer ciencia" (p. 40). De acuerdo con Hirsch y Navia (2018), la ética - en cuanto sea un sistema de códigos morales-permite a los investigadores apreciar las consideraciones estipuladas en el contexto del respeto, la consideración y el amor al prójimo, razón por la cual realizar investigaciones con personas exige la actuación ética y moral.

e) Perfil de publicación, permanencia y visibilidad

Una trilogía imprescindible para la institución universitaria y el docente investigador la constituye la publicación, la permanencia y la visibilidad. Al docente investigador se le exige la notoriedad académica, la evidencia verificable, y la publicación de artículos científicos. Asimismo, se le exige poseer estudios de posgrado (maestrías y doctorados), según la apreciación de Rodriguez-Morales et al. (2016). No solamente se le exige publicar, sino mantenerse vigente y visible para la comunidad científica. Estos tres elementos necesarios, exigidos e ineludibles. Los tres constituyen la carta de presentación del docente investigador; mejor dicho, su perfil académico. En términos coloquiales, el docente investigador "siempre está de moda".

Analizando el contexto de la investigación en Perú y Colombia, RodriguezMorales et al., 2016) suscriben que "la comunidad científica internacional reconoce que el calificativo de investigador se refleja a través de evidencia verificable: artículos en publicaciones científicas disponibles en revistas indexadas y en bases de datos internacionales (ISI/WoK, Medline/PubMed, Scopus)" (p. 256). Las publicaciones científicas también poseen determinadas exigencias. Los artículos científicos son publicados en revistas internacionales, las cuales satisfacen las condiciones y las exigencias académicas, profesionales y técnicas, válidas para todo el proceso de la 
investigación. De igual manera, a fin de lograr la notoriedad y visibilidad de las publicaciones y sus respectivos autores, es pertinente considerar las determinadas políticas institucionales, los procesos enseñanza-aprendizaje, las condiciones académicas de las investigaciones, y el establecimiento de redes y la difusión permanente (Lovera, 2016).

Aunque en las últimas décadas las universidades han incrementado los repositorios académicos-favoreciendo de esta manera la investigación $y$, por añadidura, a sus protagonistas-, los investigadores deben ser son los principales promotores y los responsables de la visibilidad, calidad, criterios, certificaciones, y los lineamientos nacionales e internacionales. En este sentido, la Universidad Nacional Mayor de San Marcos está impulsando Cybertesis Perú, un repositorio de tesis de dicha universidad, con el propósito de darles visibilidad e interoperabilidad (Cerda-Cosme, 2016).

¿Qué más decir de las publicaciones científicas en este aspecto? Las publicaciones científicas son indicadores importantes del conocimiento científico, generando incalculables beneficios a corto, mediano y largo plazo (Pulido-Medina et al., 2017). Los docentes investigadores y las universidades siempre obtienen respaldo y reconocimie nto de la comunidad académica y el Estado; ambos no prescinden de las evaluaciones, reconocimientos y premiaciones.

Por otro lado, la imagen del docente investigador posee una visibilidad interna y externa, reflejando la emancipación del docente y de la investigación, superando larga y extensamente la "falta de preparación y formación para la investigación" (Santos-Lopez, 2017). Las bibliotecas de la universidad generan y construyen la visibilidad de la investigación. Estas brindan apoyo durante los procesos de enseñanza-aprendizaje y la investigación, generando fácil acceso a los repositorios de las investigaciones (ArriolaNavarrete, 2017).

\section{f) Perfil de especialista en su disciplina}

Por otro lado, el perfil del investigador corresponde a cada disciplina, a cada especialidad. Por ejemplo, el médico trabaja en un "perfil asistencial, esencia indiscutible de la figura del posgrado correspondiente a la especialización médica" (Corona-Martínez, 2018). Los perfiles varían por la especialidad, por la disciplina, por las líneas de investigación. Por ejemplo, el perfil de los docentes investigadores en las disciplinas de la matemática, la física, la química, la biología, la medicina, etc., no tienen el mismo 
perfil; tampoco tienen el mismo perfil los docentes investigadores en literatura, antropología, sociología, filología, historia, administración, educación, contabilidad, ingeniería, arquitectura. Es decir, cada disciplina exige la presencia de su propio perfil de investigador. La correspondencia es inevitable, ineludible, imprescindible, contribuye para los éxitos de la investigación y sus respectivas calificaciones.

\section{Cómo fortalecer el perfil del docente investigador}

a) Mediante la concurrencia del investigador experto

Para Fontaines-Ruiz, Carhuachín-Marcelo y Tusa-Jumbo (2018), el perfil y las competencias investigativas en los investigadores noveles se forman con la presencia, la orientación, las competencias y la experiencia de los consolidados. El investigador experto genera "la construcción del investigador novel". Significa el encuentro de dos generaciones: la de los experimentados y la de los noveles. Implica el encuentro del maestro y los discípulos. El experto equivale a maestro, a formador, y discipulador; en cambio, el novel, se refiere al estudiante, discípulo, aprendiz. La rotura del binomio maestro-discípulo puede producir un estancamiento en el crecimiento y desarrollo de las personas y las instituciones. En este sentido, Antón-Chávez (2020) se ocupa de las orientaciones didácticas con el propósito de materializar la formación de investigadores noveles, generando ventajas para las universidades en los procesos de investigación.

b) Mediante la corrección de las deficiencias

En el ámbito mexicano, Maisterrena-González (2018) afirma que "en el área de ciencias sociales como en otras disciplinas científicas, no se cuenta con la formación y los recursos materiales y económicos suficientes para crear y producir"; por otro lado, asevera que "es fundamental una formación de investigadores más completa: se deben confrontar las deficiencias científico-tecnológicas y materiales-formativas que impidan su desarrollo", así también "es necesaria la colaboración de la academia, las empresas y el gobierno, actores que hacen viable que se impulse el conocimiento mediante recursos económicos, y a su vez, que el conocimiento contribuya a una mejor economía del país" (p. 86).

Se construye la imagen y el perfil del docente investigador generando espacios de reflexión, corrección y cuestionamientos a las deficiencias encontradas y reveladas en la formación, en la determinación de los recursos, en la falta de la colaboración de las 
personas y las instituciones comprometidas con la investigación. Hay que dedicar tiempo y recursos de manera constante para mejorar este perfil.

c) Mediante la legislación de los estados

En ciertos países, el perfil y las competencias de los investigadores son regulados; la legislación de cada país lo determinan, determina la naturaleza, las dimensiones, los niveles, las características; se supone que los procesos de la enseñanza-aprendizaje y los programas académicos de los doctorados, contribuyen a la descripción y logro del perfil del egresado; en este contexto, se entiende que la legislación de los estados regula la "formación excelente de las personas investigadoras"; también se norman y regulan la formación y desarrollo de las competencias de los investigadores (Villardón-Gallego y Villarejo-Carballido, 2018). Por ejemplo, las competencias básicas del investigador son:

- CB1. Comprensión sistemática de un campo de estudio y dominio de las habilidades y métodos de investigación relacionados con dicho campo.

- CB2. Capacidad de concebir, diseñar o crear, poner en práctica y adoptar un proceso sustancial de investigación o creación.

- CB3. Capacidad para contribuir a la ampliación de las fronteras del conocimiento a través de una investigación original.

- CB5. Capacidad de realizar un análisis crítico y de evaluación y síntesis de ideas nuevas y complejas.

- CB6. Capacidad de comunicación con la comunidad académica y científica y con la sociedad en general acerca de sus ámbitos de conocimiento en los modos e idiomas de uso habitual en su comunidad científica internacional.

- CB7. Capacidad de fomentar, en contextos académicos y profesionales, el avance científico, tecnológico, social, artístico o cultural dentro de una sociedad basada en el conocimiento (Villardón-Gallego y Villarejo-Carballido, 2018, p.159).

d) Mediante la formación investigativa

En México, Flores-Osorio (2018) estudia el panorama de la formación de los investigadores en la universidad. Para él, la formación de investigadores presenta un sinnúmero de retos, contradicciones, inconsistencias, encontrados en los contenidos programáticos, los objetivos, los perfiles de los egresados; las investigaciones se han constituido en hábitos, dogmas; son reiterativas, presentan repeticiones tradicionales; desgraciadamente, los métodos y las técnicas han sustituido la reflexión epistemológica, se desarrollan al margen de la filosofía de la ciencia; el método es tomado como un "antídoto", no despierta la crítica inteligente y alturada sobre la producción de teorías. Frente a esta situación, los estudios de posgrado (maestrías y doctorados) tienen la 
obligación de realizar doble función: formación profesionalizante y la formación en investigación.

Esta censura construye el aliento de resaltar que el perfil del docente investigad or se establece, se determina mediante la formación investigativa en el ámbito de la universidad, cuyos responsables directos son las autoridades y los docentes universitarios. La formación investigativa se inicia durante los primeros años de estudios de pregrado, motivando y estimulado el compromiso con la investigación; la consolidación de esta formación se materializa durante los estudios de las maestrías y doctorados.

e) Mediante la asistencia a eventos de investigación

Muñoz-Basols, Rodríguez-Lifante y Cruz-Moya (2017) aprecian que el investigador debe tener un perfil que revele la asistencia a eventos de investigación, cuyas contribuciones aporten al desarrollo de la comunidad y a las publicaciones periódicas, a fin de materializar la presencia en redes sociales, para atender la necesidad de construir su perfil de investigador, entre otros. Sin lugar a dudas, las convocatorias, las concurrencias, las organizaciones de eventos científicos, constituyen espacios donde se encuentran los investigadores, aunque muchas veces los costos sean excesivos; la construcción de este perfil implica tiempo, energía y dinero; es inapelable la asistencia de los docentes investigadores en la condición de conferenciantes, de expositores, de panelistas; éstos aprovechan más la calidad de los eventos, porque sus condiciones y motivaciones tienen otro nivel; sus expectativas son mayores, también sus aprendizajes. Pérez-Díaz, López-García, y Buendía-Espinosa (2019) argumentan que los congresos académicos constituyen espacios donde se forma a los investigadores, integrando de esta manera actores y prácticas diversas, cultivando aptitudes, actitudes, habilidades y valores.

f) Mediante la valoración del perfil de investigador

La investigación es compleja y muy diversificada, por las especialidades, la temática, las líneas de investigación, la metodología, los enfoques, los tipos, etc. En el escenario de la investigación en México y Brasil, Larios-Gómez (2019) suscribe dos preguntas: “¿Cuáles son las características científicas de las revistas en México y Brasil? y ¿Cuál es el perfil de los investigadores en administración en México y Brasil?” El perfil del investigador queda determinado por la producción científica, la línea de investigación del especialista, publicación en revistas de calidad científica, formación especializada en investigación, doctores en investigación. 
Es decir, el perfil del docente investigador también se determina estableciendo los criterios del perfil, las exigencias establecidas y atribuidas a los investigadores; dicho de otro modo, si no existen publicaciones y difusiones de las investigaciones, no existe un perfil investigador exigido, reclamado y demandado por la sociedad científica y las instituciones académicas.

\section{g) Mediante la intervención del Estado}

¿Cómo y en qué medida interviene el Estado en la investigación? Esta necesita infraestructura y equipamiento, cuya responsabilidad logística debe provenir de las universidades y del Estado (Luchilo y Guber, 2007). La falta de integración de estos elementos produce consecuencias gravitantes, incalculables, insospechadas, irreparables, dejando al margen el crecimiento y desarrollo de las personas y las instituciones. Para Toro-Huamanchumo et al. (2017), la universidad y el financiamiento correspondiente permiten el desarrollo de proyectos de investigación, estimulan la presencia del investigador, despliegan la producción científica.

La universidad y el Estado registran promesas incumplidas (Cuenca y Reátegui, 2016). La intromisión del Estado, sin la pericia requerida, da supremacía al sistema legal, jurídico, en nombre "de la defensa y protección de los derechos fundamentales" (NoriegaEgas, 2017). Esta radiografía inversa de la intervención del Estado en los procesos de la investigación, se debe concebir en positivo, dejando el mensaje de que el Estado y su participación coronarán los éxitos de la investigación, el logro del perfil del docente investigador, cuyos resultados no dejarán al margen el crecimiento y desarrollo de las instituciones y de las naciones. Sobre este aspecto, de acuerdo con las apreciaciones de Flores-Osorio (2018),

La investigación y la Formación de investigadores en México se desarrolla bajo parámetros administrativo-contables postulados por el Estado mexicano a través de organis mos como el Consejo Nacional de Ciencia y Tecnología (CONACyT), la Secretaría de Educación Pública (SEP) y por la Asociación Nacional de Universidades e Instituciones de Educación Superior (ANUIES). (p.48).

h) Mediante la generación de espacios y condiciones iguales para todos

En este espacio cabe la presencia de la interrogante: ¿existe igualdad de competencias y condiciones para todos? La primera idea que nace es que la investigac ión revela un conjunto de problemas desiguales y complicados, no son uniformes, tampoco 
comunes. Los artículos publicados en google académico no están publicados en revistas indizadas, solamente aparecen en español, no aparecen en la versión de inglés. Son reducidas las publicaciones de los asesores de tesis, por la falta de experiencia y calidad en la investigación; las publicaciones no tienen la rigurosidad ni calidad, porque demandan y exigen los revisores expertos (Atamari-Anahui, Sucasaca-Rodríguez y Marroquin-Santa Cruz, 2016). La imagen de la universidad se exhibe en espacios y plataformas de investigación; por ejemplo, en la base de datos de SCOPUS, generado impacto institucional y a nivel internacional, cuyos datos e información alimentan el SCImago-Journal y Country-Rank (SJR). En este espacio sobresalen los países más desarrollados, los costos son excesivos. Las posiciones y ubicaciones, en el contexto mundial, la encabezan los Estados Unidos, China, Reino Unido, Alemania, Japón. En Latinoamérica, Brasil es el país con mayor producción, quedando en la decimoquinta ubicación a nivel mundial, luego aparecen México, Argentina y Chile. El Perú aparece en la octava posición, en la región; a nivel mundial, en septuagésima posición. Aparecen con mayor presencia en el orden: medicina, agricultura, bioquímica, genética, biología molecular, durante el año 2014. Las universidades de mayor presencia son: Universidad Peruana Cayetano Heredia, Universidad Nacional Mayor de San Marcos, Pontificia Universidad Católica del Perú, periodo 2015 (Gonzales-Saldaña et al., 2017).

En el panorama de la investigación, ¿cómo están las bibliotecas de ciencias sociales? ¿Qué dicen los investigadores sobre las mismas? ¿Tienen presencia en los espacios de la investigación? ¿Sirven para la acreditación de las universidades? Sobre estas cuestiones, Corda (2017) afirma que de estas bibliotecas casi nunca se habla, no son renombradas, tampoco tratadas en las bibliografías, ni en las actividades académicas de las "élites" de investigación. Sin embargo, estas bibliotecas son bastante visitadas y consultadas, las visitan e investigan solamente quienes pertenecen a las ciencias sociales.

Esto exige la presencia de los investigadores con oportunidades iguales para todos. Reclaman la construcción del perfil del docente investigador de las "disciplinas relegadas", en las condiciones y las dimensiones que tales disciplinas lo exigen y determinan, teniendo también la oportunidad de dejar su presencia en los espacios de la difusión de las investigaciones correspondientes a las ciencias puras.

i) Mediante el cultivo de una actitud positiva de los estudiantes

El desarrollo del perfil docente no solamente depende del docente, también depende del estudiante, especialmente en su actitud. Pocos estudiantes universitarios tienen una 
percepción positiva sobre la investigación realizada en la universidad. El 30\% valora la investigación; el 60\% califica deficiente la capacitación de los profesores, prefieren mayor nivel cognitivo, lecturas críticas, redacción de artículos, procesos de publicación, búsqueda bibliográfica (Molina-Ordóñez, Huamaní y Mayta-Tristán, 2008).

Por oposición y contraste, lo referido queda en el plano de lo negativo; significa, entonces, que es necesaria la actitud positiva del estudiante sobre la investigación; eliminada esa valla de la desconexión con la investigación.

La indiferencia y la apatía ha dejado ausencia de los estudiantes en las publicaciones científicas, es una respuesta a la falta de preparación y formación, en los estudios de pre grado, dejando al margen el hábito y cultura de investigación, la experiencia y el criterio científico (Bendezú-Quispe et al, 2015). Castro-Rodríguez, Sihuay-Torres y PerezJiménez (2016) declaran que los estudiantes han realizado una publicación científica muy baja, los estudiantes perciben sus conocimientos ubicados en la escala de regular, sobre las metodologías, la redacción científica y la búsqueda de información científica. Se requiere experiencias, criterio científico, hábitos y cultura en investigación.

Según Alarco, Changllio-Calle y Cahuana-Salazar (2017), la motivación en la investigación experimenta un descenso mientras los estudiantes avanzan semestres de estudios. Durante los primeros ciclos, el interés es muy notorio, es mayor en las mujeres que en los hombres, en la redacción de los artículos científicos. Jurado-Vargas (2017), en un estudio con 100 estudiantes, muestra que el 66\% muestra una actitud medianamente favorable. Las actitudes hacia la investigación no son adecuadas, generando una desventaja mayúscula para la universidad; la enseñanza y la práctica investigativa presentan grandes dificultades, en la calidad y en la cantidad, por la heterogeneidad de las condiciones, los factores y los sujetos; la investigación científica en el pre grado revela una crisis, por la baja producción y por la calidad (Mercado-Rey, 2018). Las habilidades científicas y las habilidades clínicas son muy importantes, de gran valor. Se constituyen en competencias para la vida profesional, se suman a las actitudes positivas y motivadoras (Dorta-Contreras, Corrales-Reyes y Fornaris-Cedeño, 2019).

\section{Conclusiones}

En el contexto universitario e investigativo, el perfil del docente investigador se ha constituido en un asunto de constante abordaje, de preocupación académica y administrativa, inclusive de gestión económica, sin dejar al margen la evaluación, la selección y el prestigio interno, nacional e internacional. Las universidades, al interior y 
al exterior de las mismas, hablan de investigación, son sintonizadas y respaldadas a través de los profesores investigadores y sus respectivos perfiles, cuyos artículos son sometidos, admitidos y publicados en revistas indexadas de investigación.

Respecto del perfil del docente investigador, se han consultado investigaciones relacionadas con las dimensiones y los procedimientos para fortalecer el perfil referido. En este sentido, se han consultado y escudrinado estudios abundantes, actualizados $\mathrm{y}$ pertinentes, logrando así una mayor cosmovisión, amplitud y claridad cognitiva y procedimental sobre el particular.

Sobre las dimensiones del perfil del docente investigador, luego de la investigación respectiva, se han determinado, entre otras, las siguientes: perfil pedagógico, perfil académico profesional, perfil de inteligencia emocional, perfil ético, perfil de publicación, permanencia y visibilidad, perfil de especialista en su disciplina.

El perfil pedagógico implica que docente investigador ha logrado sus competencias, capacidades y habilidades pedagógicas; ha asumido un notable compromiso con la enseñanza de la investigación, enseñando la construcción correcta, apropiada y estandarizada del marco teórico, de la operación de las variables, de la elaboración de los instrumentos, de los análisis teóricos y estadísticos, de la presentación de los informes. Este perfil significa la presencia de un pedagogo de la investigación científica, quien conoce y aplica los lineamientos y los principios pedagógicos, quien tiene respaldo en la metodología pedagógica; practica una enseñanza sistematizada, facilita con eficacia los aprendizajes de los estudiantes, quienes se constituyen en discípulos de la investigación.

Por su parte, el perfil académico profesional revela cuánto el docente investigador se ha logrado académica y profesionalmente; permite observar qué proyecta, qué imagen de la institución donde se ha formado académicamente y cómo se proyecta hacia el futuro profesional, si revela confianza y seguridad para los demás; nos da la perspectiva de cómo la universidad (la academia) deja al profesional en el espacio y en el escenario de la investigación; la investigación y la academia se encuentran en el perfil académico profesional del docente investigador. La universidad respalda y construye el perfil académico profesional, dejando la cosecha de las competencias, las capacidades y las habilidades comprometidas con el mundo de la investigación.

De otra manera, la inteligencia emocional, en la perspectiva de dimensión de perfil del docente investigador, produce y cuantifica el razonamiento válido y eficiente; cultiva y fortalece la vida emocional saludable; regula las emociones, no solamente las recibe; facilita un desenvolviendo muy positivo y empático durante las relaciones interpersonales 
y los procesos de investigación, comprendiendo y entendiendo plenamente a todos los actuantes durante los procesos de la comunicación, la inter e intra relación.

El docente investigador revela su propio perfil ético, durante los procedimientos de la investigación, tiene que ajustar sus actuaciones investigativas, a las perspectivas y a los consentimientos éticos y morales, dejando, para la observación de los demás, su conducta investigativa que revela trasparencia, equidad, justicia, honestidad, integridad académica, científica y profesional. La conducta del docente investigador no rompe los parámetros universales: éticos y morales; sabiamente aprecia lo que significa trabajar la ciencia, sin vulnerar la integridad y la salud de las personas y de los animales, con la cultura y los valores nacionales.

Se ha trazado el perfil de publicación, permanencia y visibilidad en el docente investigador, subrayando que a éste se le exige la permanencia de su visibilidad, la cual implica además la evidencia verificable, las publicaciones de los artículos científicos en revista indexadas, nacionales e internacionales, respetando los estándares y los protocolos internacionales de la investigación científica, cuyas exigencias son académicas, técnicas y profesionales. Este perfil del docente investigador hace conciencia de que las publicaciones científicas son encaminadas de acuerdo con las políticas institucionales, en el marco de las condiciones académicas determinadas y referidas a los repositorios, las revistas, las bibliotecas, entendiendo que la visibilidad (interna y externa), las certificaciones, la calidad y la imagen no solamente le corresponden al investigador, sino a la institución que patrocina.

En torno del perfil de especialista en su disciplina, el docente investigador adquiere la postura de un especialista en la materia académica; es un investigador en su propia línea, pues las disciplinas del saber humano obligan la presencia y permanencia de especialistas en las respectivas materias y disciplinas. Los criterios, metodologías, procedimientos, instrumentos, etc., no son los mismos para todas las disciplinas; por ejemplo, las matemáticas, las ciencias médicas, las ciencias biológicas, las ciencias sociales. Cada disciplina y cada especialidad requieren un perfil exclusivo del docente investigador, para garantizar la fortaleza y la calidad de la investigación.

En relación con la determinación y selección de los procedimientos para fortalecer el perfil del docente investigador, se concluye que dicho perfil será fortalecido mediante la concurrencia del investigador experto, mediante la corrección de las deficiencias, la legislación de los estados, la formación investigativa, la asistencia a eventos de investigación, la valoración del perfil de investigador, la intervención del Estado, la 
generación de espacios y condiciones iguales para todos, el cultivo de una actitud positiva de los estudiantes.

En este sentido, resulta imprescindible la concurrencia del investigador experto, quien consolida las competencias de los investigadores jóvenes y noveles; así, el investigador experto genera "la construcción del investigador novel". Establece una necesaria vinculación de dos generaciones, las cuales muchas veces aparecen y actúan divorciadas y en oposición; las dos se alimentan recíprocamente; este perfil significa el encuentro del maestro y el discípulo en el mismo escenario: la investigación.

El perfil de docente investigador se fortalece con la práctica, con la participación en los procesos de aprendizaje y corrección, con el propósito de enmendar y superar las deficiencias, errores, equívocos, debilidades, desaciertos. Los investigadores intelige ntes y desarrollados reciben, respetan y experimentan los procesos de formación investigativa, razón por la cual se sustenta, con verdad y claridad, la formación de investigadores noveles durante los estudios universitarios, convocando y construyendo espacios apropiados para la reflexión, el cuestionamiento y la corrección de las deficiencias, las debilidades y los desaciertos, en forma sabia, pertinente y oportuna.

En este proceso, son necesarias las múltiples intervenciones. La participación del Estado también fortalece el perfil del docente investigador. Este perfil requerido es fortalecido mediante las respectivas legislaciones de los Estados, los cuales, a través de los poderes políticos, norman y regulan mediante las leyes pertinentes, marcando los derroteros de la actuación de los investigadores, los requerimientos de la calidad, la formación y los perfiles de los investigadores.

El docente y su perfil, durante los años iniciales, necesitan formación investigativa, la cual se realiza en los claustros universitarios, en un universo de muchos y constantes retos, en medio de las experiencias llenas de contradicciones mayúsculas e inconsistencias por la complejidad y diversidad de sujetos, objetos y contenidos. La formación se realiza en torno de las metodologías, las técnicas, la crítica inteligente, la producción de las teorías, el compromiso investigativo, la consolidación de los paradigmas investigativos.

Para el docente investigador, la asistencia a eventos de investigación se ha constituido en una obligación moral y académica. La asistencia a estos eventos puede generar crecimiento y desarrollo de las capacidades y habilidades, gerenciamiento de nuevas posibilidades y el fortalecemiento del perfil del docente investigador, amplía su 
cosmovisión, destruye los paradigmas errados de la investigación, además que construye y amplía la red de los investigadores.

En este sentido, ¿cómo queda la valoración del perfil de docente investigador? Se espera que la consolidación y permanencia de esta valoración, con la intervención de la universidad, del Estado y de las instituciones comprometidas, logren alcanzar los objetivos y las metas de la investigación científica, estableciendo los respectivos marcos legales, económicos y éticos. De esta forma, los perfiles de los docentes investigadores necesitan ser valorados y difundidos, con el propósito de motivación y reconocimiento.

Por otro lado, los espacios y las condiciones no son iguales para todos. Los escenarios no son los mismos, tampoco iguales para todos. Los problemas son desiguales, las soluciones son aun disímiles, los lenguajes también son desiguales, así como la calidad y su rigurosidad llegan a todos, por la edad, el género, el país, la región, la especialidad o disciplina, la universidad. En este contexto, quedan la palabra y la voz reclamando espacios, condiciones y evaluaciones equitativas, justas y oportunas para todos, para todas las disciplinas del campo científico y pedagógico.

Finalmente, en el espectro del perfil del docente investigador, no queda al margen el cultivo de una actitud positiva de parte de los estudiantes, quienes tienen oportunidad de integrar el contexto académico de la investigación, garantizando su crecimiento y desarrollo de sus competencias y habilidades. De esa manera, se garantiza el futuro de la investigación, de las instituciones y de los Estados.

\section{Referencias}

Alarco, J. J., Changllio-Calle, G. y Cahuana-Salazar, M. (2017). Investigación en pregrado: interés según sexo y ciclo académico. Educacion Médica, 18(1), 67-73. https://doi.org/10.1016/j.edumed.2016.04.004

Antón-Chávez, A. P. (2020). Orientaciones didácticas para un plan de formación de investigadores noveles. Revista Conrado, 16(72), 298-305. Recuperado de https://orcid.org/0000-0002-2939-1346

Arriola-Navarrete, Ó. (2017). Centro de Recursos para el Aprendizaje y la Investigación (CRAI): entorno dinámico de servicios. Bibliotecas y Archivos: Órgano de la Escuela Nacional de Biblioteconomía y Archivonomía, 2(4), 16-29. Recuperado de http//hdl.handle.net/10366/119110\%5Cnhttp://gredos. usal.es/jspui/handle/10366/1 19110

Atamari-Anahui, N., Sucasaca-Rodríguez, C. y Marroquin-Santa Cruz, J. A. (2016). 
Publicación científica de asesores de tesis de pregrado en una escuela de medicina de Cusco, Perú (Carta al editor). Cusco, Perú: Asociación Científica de Estudiantes de Medicina Humana (ASOCIEMH-CUSCO). https://oi.org/10.1016/j.riem.2016.05.002

Bendezú Quispe, G., Hurtado Horta, S., Medina Saravia, C. E. y Aguilar León, P. (2015). Apreciación sobre capacitación en investigación y publicación científica en estudiantes universitarios. Investigación en Educación Médica, 4(13), 50-51. https://doi.org/10.1016/S2007-5057(15)72169-1

Canizales-Rivas, G. P. y Vijil-Mejía, J. (2015). Necesidades y características de los docentes que enseñan investigación en el centro universitario regional del Valle del Aguán (Curva) y en el Centro Universitario regional del Litoral Atléntico (Curla) (Tesis de maestría), Universidad Nacional Autónoma de Honduras, Tegucigalpa, Honduras. Recuperado de http $/ / w w w . t z i b a l n a a h . u n a h . e d u . h n / h a n d l e / 123456789 / 5930$

Castro-Rodríguez, Y., Sihuay-Torres, K. y Perez-Jiménez, V. (2016). Producción científica y percepción de la investigación por estudiantes de odontología. Educación Médica, 1-4. https://doi.org/10.1016/j.edumed.2016.11.001

Cerda-Cosme, R. P. (2016). Cybertesis Perú, evaluación del repositorio de tesis de la Universidad Nacional Mayor de San Marcos (Tesis de licenciatura), Universidad Nacional Mayor de San Marcos, Lima Perú. Recuperado de https://cybertesis.unmsm.edu.pe/bitstream/handle/20.500.12672/5795/Cerda_cr.pdf ?sequence $=1 \&$ isAllowed $=\mathrm{y}$

Corda, M. C. (2017). De las que casi nunca se habla: las bibliotecas de investigación. La trayectoria de la Biblioteca de Ciencias Sociales "Enzo Faletto" de FLACSO, Argentina. Boletín Electrónico ABGRA, 9(3), 1-11. Recuperado de http//sedici.unlp.edu.ar/bitstream/handle/10915/89232/Documento_completo.8044 .pdf? sequence $=1 \&$ is Allowed $=\mathrm{y}$

Corona-Martínez, L. A. (2018). La formación de médicos investigadores. A propósito del día de la Ciencia en Cuba, Medisur, 16(3), 488-491. Recuperado de http $/ /$ scielo.sld.cu/pdf/ms/v16n3/ms17316.pdf

Cuenca, R. y Reátegui, L. (2016). La (incumplida) promesa universitaria en el Perú. Lima, Perú: Instituto de Estudios Peruanos. Recuperado de http//biblioteca.clacso.edu.ar/Peru/iep/20170328054601/pdf_1498.pdf\%0Ahttp://r epositorio.iep.org.pe/handle/IEP/9 
Dorta-Contreras, A. J., Corrales-Reyes, I. E. y Fornaris-Cedeño, Y. (2019). Producción científica estudiantil en las revistas biomédicas indexadas en SciELO Cuba 2015 y 2016. Investigación en Educación Médica, 8(30), 29-40. https://oi.org/10.22201/facmed.20075057e.2019.30.1785

Flores-Osorio, J. M. (2018). Retos y contradicciones de la formación de investigadores en México Pesquisadores no México Challenges and contradictions at the training the Researchers in Mexico. Educar em Revista, Curitiba, Brasil, 34(71), 35-49. https://doi.org/10.1590/0104-4060.62554

Fontaines-Ruiz, T., Carhuachín Marcelo, A. I. y Tusa Jumbo, F. (2018). Competencias formativas de los investigadores noveles según los investigadores consolidados. Educación, XXVII(53), 107-127. Recuperado de https://doi.org/10.18800/educacion.201802.007

Gonzales-Saldaña, J., Chavez-Uceda, T., Lemus-Arteaga, K., Silva-Ocas, I., GalvezOlortegui, T. y Galvez-Olortegui, J. (2017). Producción científica de la facultad de medicina de una universidad peruana en SCOPUS y Pubmed. Educ Med., 1-8. https://doi.org/10.1016/j.edumed.2017.01.010

Hernández-Herrera, C. A. y Ramos-Mendoza, J. R. (2018). La inteligencia emocional y la práctica docente en profesores investigadores. RIDE Revista Iberoamericana para la Investigación y el Desarrollo Educativo, 9(17), 1-29. https://doi.org/10.23913/ride.v9i17.387

Hirsch, A. y Navia, C. (2018). Ética de la investigación y formadores de docentes Research Ethics and Teacher Trainers. Revista Electrónica de Investigación Educativa, 20(3), $1-10$. https://doi.org/https://doi.org/10.24320/redie.2018.20.3.1776

Hortiguiela, D., González, G. y Hernando, A. (2017). Valoración del investigador sobre los códigos éticos en el ámbito científico. Perfiles Educativos, XXXIX(155), 38-50. Recuperado de http $/ /$ www.scielo.org.mx/pdf/peredu/v39n155/0185-2698-peredu39-155-00038.pdf

Jurado-Vargas, S. C. (2017). Actitudes hacia la investigación en estudiantes de enfermería de la UNMSM, 2016 (Tesis de Licenciatura), Universidad Nacional Mayor de San Marcos, Lima, Perú. Recuperado de https://cybertesis.unmsm.edu.pe/bits tream/handle/20.500.12672/6149/Jurado_vs.p df? sequence $=1$

Larios-Gómez, E. (2019). La investigación científica, las revistas y el perfil del 
investigador en las ciencias de gestión: México-Brasil. Cuaderno Técnico de Administração Contemporânea, 1(1), 51-68. Recuperado de https://webserver1.fumep.edu.br/ojs/ojs-2.4.8-5/ind ex.php/CTAC/article/view/5168

Lovera, D. F. (2016). Políticas, enseñanza, investigación, redes y difusión ambiental. Revista del Instituto de Investigación, FIGMMG-UNMSM, 19(38), 129-135. Recuperado de https://re vistasinvestigacion.unmsm.edu.pe/index.php/iigeo/article/view/13579/119 90

Luchilo, L. y Guber, R. (2007). La infraestructura para la investigación universitaria en la Argentina. Educación Superior y Sociedad, 1(1), 114-131. https://doi.org/http://ess.iesalc.unesco.org.ve/ess3/index.php/ess/article/view/26

Maisterrena-González, M. (2018). La inserción laboral de los egresados del sistema universitario con perfil de investigador en México. Un breve análisis de factores históricos y problemas institucionales académicos. PROMETEICA - Revista de $\begin{array}{llll}\text { Filosofía } \quad y & \text { Ciencias, } & \text { 78-88), }\end{array}$ https://doi.org/https://doi.org/10.24316/prometeica.v0i16.211

Mercado-Rey, M. R. (2018). Actitudes hacia la investigación en los estudiantes de la carrera de Medicina Humana de la Universidad Peruana Los Andes. Educacion Medica, 1-4. https://doi.org/10.1016/j.edumed.2017.10.012

Molina-Ordóñez, J., Huamaní, C. y Mayta-Tristán, P. (2008). Apreciación estudiantil sobre la capacitación universitaria en investigación: estudio preliminar. Rev Peru Med Exp Salud Publica, 25(3), 325-329. https://doi.org/10.17843/RPMESP.2008.253.1283

Muñoz-Basols, J., Rodríguez-Lifante, A. y Cruz-Moya, O. (2017). Perfil laboral, formativo e investigador del profesional de español como lengua extranjera o segunda (ELE / EL2): datos cuantitativos y cualitativos. Journal of spanish language teaching, 4(1), 1-34. https://doi.org/10.1080/23247797.2017.1325115

Noriega-Egas, C. R. (2017). La excesiva intervención del juez constitucional en el diseño de política de reforma universitaria en el Perú. (Tesis de maestria), Pontificia Universidad Católica del Perú, Lima, Perú. Recuperado de http//tesis.pucp.edu.pe/repositorio/bitstream/handle/20.500.12404/8565/NORIEG

A_CARMEN_reforma constitucional_reforma universitaria.pdf? sequence $=1 \&$ is Allowed $=y$ 
Pérez-Díaz, A. B., López-García, J. C. y Buendía-Espinosa, A. (2019). El congreso académico como espacio para la formación de investigadores. Revista Mexicana de Investigación Educativa, 24(83), 1109-1134. Recuperado de http //www.scielo.org.mx/pdf/rmie/v24n83/1405-6666-rmie-24-83-1109.pdf

Pulido-Medina, C., Hamon-Rugeles, D., López-Ramírez, E., Quimbayo-Cifuentes, A. F. y Mejía, C. R. (2017). Publicación científica entre los directivos de la Asociación Científica de Estudiantes de Medicina de Colombia: características y factores asociados. Rev. Fac. Med, 65(4), 553-557. https://doi.org/10.15446/revfacmed.v65n4.60489

Rodriguez-Morales, A. J., Díaz-Vélez, C., Gálvez-Olórtegui, T., Benites-Zapata, V. y Gálvez-Olórtegui, J. (2016). ¿Cuál debería ser el perfil de quien se denomine investigador en Colombia y Perú? Carta al editor. How should be the profile of who called as researcher in Colombia and Peru? Acta Med Per., 33(3), 256-258. https://doi.org/http://www.scielo.org.pe/pdf/amp/v33n3/a19v33n3.pdf

Santos-Lopez, A. (2017). Flashes de emancipación en la actuación docente e investigación universitaria de los miembros del sistema nacional de investigadores (SNI). Roteiro, Joaçaba, 11-36. https://doi.org/http://dx.doi.org/10.18593/r.v42i1.11547

Toro-Huamanchumo, C. J., Arce-Villalobos, L. R., Gonzales-Martínez, J., MelgarejoCastillo, A. y Taype-Rondán, Á. (2017). Financiamiento de la investigación en pregrado en las facultades de medicina peruanas. Ga Sanit, 31(6), 541-542. Recuperado de http://dx.doi.org/10.1016/j.gaceta.2016.06.009

Villardón-Gallego, L. y Villarejo-Carballido, B. (2018). Percepciones de investigadores en formación respecto al desarrollo de competencias. Revista Digital de Investigación en Docencia Universitaria, 12(2), 157-179. Recuperado de http $/ /$ www.scielo.org.pe/pdf/ridu/v12n2/a08v12n2.pdf 